\title{
The IR Characteristics Modeling and Simulation of the HEO Target
}

\author{
Tao Dongxing ${ }^{1, a}$, Lin Boying ${ }^{1}$, Du Peng ${ }^{1}$, Bi Yanqiang ${ }^{1}$, Shang Yonghong ${ }^{1}$, Li Xiyuan ${ }^{1}$ and Wang Jing ${ }^{1}$ \\ ${ }^{1}$ Beijing Institute of Spacecraft Environment Engineering, Beijing 100094, China
}

\begin{abstract}
Space target infrared (IR) characteristics model can be used to design space target detection sensor, generating simulation data to validate the data processing algorithms, such as target detection and tracking. In the work, a HEO target IR characteristics model is built. The model consists of the geometry module and the IR radiometric module. Unlike the traditional space IR model, the temperature of cabin inner is used as the simulation origin. Using the model, the irradiance spectra of HEO target are calculated for different target temperature. With the calculation results, the main wavelength range for HEO detection is analyzed. Using the equvalent temperature, the work also designs a simulative target, which has the similar IR characteristics of a HEO target. The simulator can be used in the ground test for the imaging sensor or target decoy.
\end{abstract}

\section{Introduction}

As a passive detection manner, IR detection has many advantages, such as the excellent concealment, adapting to day and night work, and the high sensitivity for the temperature difference between target and background [1]. So that, IR detection has been widely developed and applied for the space target detection. Now, there has abundant work dedicated to the modeling and digital simulation of the space target IR characteristics [2, 3]. The digital simulators can provide the IR radiation of the target for the given orbit and attitude, and it could be used to assist the detection sensor design and provide simulation data for processing method development. The traditional modeling methods use the target surface temperature as the simulation origin to calculate the IR radiation [4]. However, the target surface temperature is unknown in the normal condition. The temperature message can be gotten is the inner cabin and the instrument. Usually, the cabin is covered by the thermal control unit and coating. Therefore, the model should use the inner cabin temperature as the simulation origin, and the heat transmission of the space target should be considered. In order to evaluate the detection ability of the developed IR imaging sensor in the ground or design decoy target for the important space target, a simulative target with the similar IR characteristics is needed. In the work, the IR characteristics modeling of the HEO target is built with the target orbit, surface material, inner cabin temperature, and attitude considered. Moreover, a simulative target with the given IR radiation is designed for the ground test.

\footnotetext{
${ }^{\mathrm{a}}$ Corresponding author : taodx2016@163.com
} 


\section{The IR characteristic modeling of the HEO target}

\subsection{Geometry module}

The body surface of space target can be expressed by a large number of facets based on computer graphics theory. All facets are convex polygons. Their shapes, sizes, and positions can be uniquely defined by their 3D coordinates and exterior normal directions in a coordinate system. The professional geometry modeling software 3DS MAX is used to build the geometry model [5]. Then the material characteristic of each facet is defined.

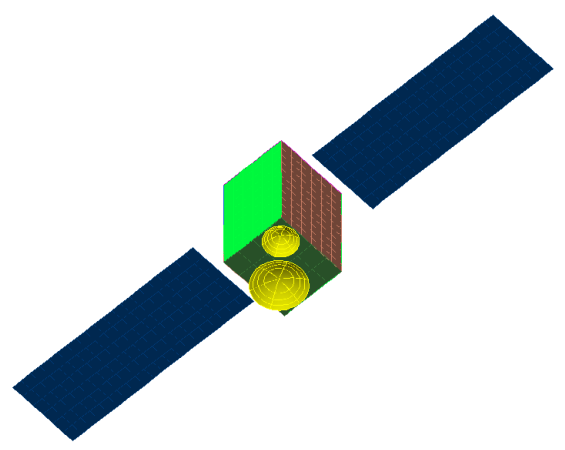

Figure 1. The sketch of the geometry configuration.

As the special equipment, each space target has the different configuration. The complex degree of the configuration determines the calculation quantity on the digital simulator. Therefore, a trade-off should be made between the simulation precise and the calculation quantity. In the work, a satellite geometry module, which consists of satellite body, two solar wings, and two antennas, is built. Figure 1 shows the sketch of the space target geometry configuration.

\subsection{IR Radiometric Module}

The IR radiation of the space target, which reaches the imaging sensor, comprises the reflected radiation and the facet-self emission radiation:

$$
L(\lambda)=L_{\mathrm{e}}(\lambda)+L_{\mathrm{r}}(\lambda)
$$

Where $L$ is the radiance the imaging sensor received, $L_{\mathrm{e}}$ is the emission part, $L_{\mathrm{r}}$ is the reflection part, and $\lambda$ represents the wavelength.

\subsubsection{Reflected irradiance of the target facet}

The radiation received by the target facet includes three parts: solar irradiance, lunar irradiance, and earth irradiance. The lunar irradiance reaches the earth satellite is very low because of the $3.8 \times 10^{5}$ kilometers of the lunar-earth distance. Therefore, the irradiance reaches the target $\left(E_{\mathrm{tr}}\right)$ is:

$$
E_{\text {tr }}(\lambda)=E_{\mathrm{s}}(\lambda)+\left[E_{\mathrm{es}}(\lambda)+E_{\mathrm{s}}(\lambda) \cos \theta_{1} \mathrm{r}_{\mathrm{e}}(\lambda)\right] \times \tau_{\mathrm{a}}(\lambda)
$$

Where $E_{\mathrm{s}}$ is the TOA (Top of Atmosphere) solar irradiance, $E_{\mathrm{es}}$ is the emission irradiance of the earth, $\theta_{1}$ is the solar altitude angle relative to nadir point, $r_{\mathrm{e}}$ is the equivalent reflectance of the earth, $\tau_{\mathrm{a}}$ is the transmittance of the atmosphere. Based on the Planck's Law [6], $E_{\mathrm{s}}$ and $E_{\mathrm{es}}$ are represented by:

$$
E_{s}(\lambda)=\frac{2 h c^{2}}{\lambda^{5} d_{s e}^{2}\left\{\exp \left[h c /\left(\lambda k_{B} T_{s}\right)\right]-1\right\}} \varepsilon_{s}(\lambda)
$$




$$
E_{e s}(\lambda)=\frac{2 h c^{2}}{\lambda^{5} d_{e t}^{2}\left\{\exp \left[h c /\left(\lambda k_{B} T_{e}\right)\right]-1\right\}} \varepsilon_{e}(\lambda)
$$

Where $h$ is the Planck's constant, $k_{B}$ is the Boltzmann's constant, $c$ is the velocity of light, $T_{\mathrm{S}}$ is the temperature of the solar surface, $T_{\mathrm{e}}$ is the temperature of the earth surface, $\varepsilon_{\mathrm{s}}$ is the emissivity of the solar surface, $\varepsilon_{\mathrm{e}}$ is the emissivity of the earth surface, $d_{\mathrm{se}}$ is the solar-earth distance, and $d_{\mathrm{et}}$ is the earthtarget distance.

The received irradiance from the earth is very low for the HEO target, and it could be ignored in the work. Then, the $L_{\mathrm{r}}$ is represented by [7]:

$$
L_{\mathrm{r}}(\lambda)=E_{\mathrm{s}}(\lambda) \mathrm{r}_{\mathrm{BRDF}}(\lambda) \cos \theta
$$

Where $\mathrm{r}_{\mathrm{BRDF}}$ is the BRDF (Bidirectional Reflectance Distribution Function) of the target surface, and $\theta$ is the solar altitude angle relative to surface facet.

\subsubsection{Emission irradiance of the target facet}

In order to get the emission irradiance of the target, the surface temperature should be calculated firstly. For the HEO target, the environment heat flux is only the solar radiation with the earth radiation ignored. Considering the conversation of energy in the heat balance condition, which is showed in the Figure 2, the surface temperature can be represented by:

$$
\alpha q=-\sigma \frac{T_{i}-T_{o}}{\delta}
$$

Where $\alpha$ is the absorptivity of the facet, $q$ is the environment heat flux, $\sigma$ is the thermal conductivity of the facet material, $\delta$ is the depth of the thermal control unit and coating, Ti and T0 are the temperature of the cabin inner and the surface separately.

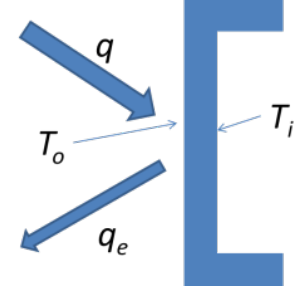

Figure 2. The sketch of the heat transmission.

The target received heat flux can be calculated when the 3D-configuration and attitude of the target is provided. Then the target surface temperature is represented by:

$$
T_{o}=\frac{\alpha q \delta}{\sigma}+T_{i}
$$

In order to improve the thermal control ability of the satellite, isothermal temperature design of the cabin is used widely. Therefore, each cabin and solar wing can be defined a uniform temperature separately, and the target emission irradiance can be calculate.

In the work, the emission characteristic of the target surface is supposed isotropic. Therefore, the Planck's Law showed in Equation (3) is used to calculate the Emission irradiance of the target $E_{\mathrm{e}}$ with the emissivity of the facet $\varepsilon_{\mathrm{t}}$ considered.

$$
E_{e}(\lambda)=\frac{2 h c^{2}}{\lambda^{5}\left\{\exp \left[h c /\left(\lambda k_{B} T_{t}\right)\right]-1\right\}} \varepsilon_{t}(\lambda)
$$

Where $T_{\mathrm{t}}$ is the temperature of the target facet. 


\subsection{HEO Target radiation characteristic}

Using the Equation (3) and (6), the irradiance target received and emission is showed in Figure 3 for different temperature.

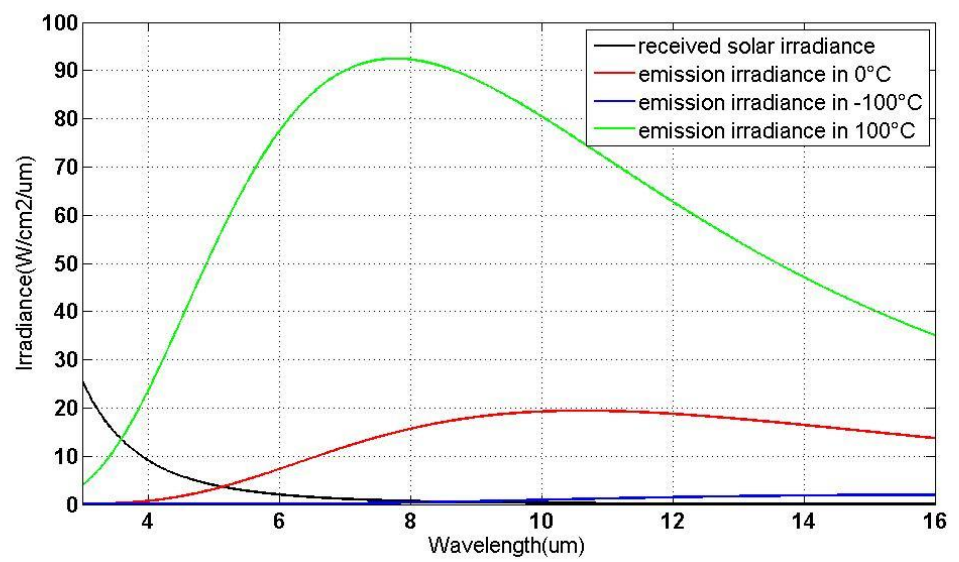

Figure 3. The received solar irradiance and emission irradiance.

It can be shown in Figure 3 that the emission irradiance is mainly distributed in the wavelength range of $3 \sim 14 \mu \mathrm{m}$. For the HEO target, the filter is usually used as the wavelength chosen device. With the sensor SRF (Spectral Responsible Function) $R_{\mathrm{s}}$ considered, the IR radiation be detected is represented by [8]:

$$
E_{d}=\frac{\int_{\lambda_{1}}^{\lambda_{2}} L(\lambda) \times \Omega \times R_{s}(\lambda) d \lambda}{\int_{\lambda_{1}}^{\lambda_{2}} R_{s}(\lambda) d \lambda}
$$

Where $\lambda_{1}$ and $\lambda_{2}$ is the response wavelength of the sensor, and $\Omega$ represents the solid angle of the sensor. With the Equation (9) considered, the ratio between received solar irradiance and the emission irradiance in Figure 3 is calculated for different temperature and wavelength band. The calculation results are showed in the Table 1.

Table 1. The ratio between received irradiance and emission irradiance.

\begin{tabular}{c|ccc}
\hline Calculation Parameter & $\mathbf{- 1 0 0}^{\circ} \mathbf{C}$ & $\mathbf{0}^{\circ} \mathbf{C}$ & $\mathbf{1 0 0}^{\circ} \mathbf{C}$ \\
\hline $\mathbf{3} \sim \mathbf{1 4} \boldsymbol{\mu m}$ & 0.3 & 4.9 & 25.0 \\
$\mathbf{8} \sim \mathbf{1 4} \boldsymbol{\mu m}$ & 4.6 & 72.7 & 283.5 \\
\hline
\end{tabular}

The target temperature will not decrease to $-100^{\circ} \mathrm{C}$, so that the main radiation of the target is the emission part. Moreover, the reflected radiation will not enter the FOV (Field of View) sometimes for the HEO target. Therefore, the emission irradiance of the target is more important for the detection sensor. The temperature of the target will not rise to $100{ }^{\circ} \mathrm{C}$, so that wavelength range of $8 \sim 14 \mu \mathrm{m}$ is the concerned only.

\section{HEO target simulator}

In the work, a HEO target simulator, which has the similar IR characteristic as the truly target, is designed. The target is in the geostationary orbit, and the attitude is earth orientation. The 3Dconfiguration is the same as the Figure 1. Two working cases of the target, which are the worst cold case and the worst hot case, are considered in the work. The worst cold case appears when the target crosses the equinox point in its early life, and the worst hot case appears when the target crosses 
solstice point in its end life. For the HEO target, the underside cabin cannot be observed all the time. And the temperature of other cabin and solar wing is showed in the Table 2. The surface materials are the MLI (Multi-Layer Insulation), the thermal control paint, the OSR (Optical Solar Reflector), and the carbon fibre envelope.

Table 2. The temperature of each cabin and the solar wing.

\begin{tabular}{c|cc}
\hline position & $\begin{array}{c}\text { The worst cold case } \\
\left({ }^{\circ} \mathbf{C}\right)\end{array}$ & $\begin{array}{c}\text { The worst hot case } \\
\left({ }^{\circ} \mathbf{C}\right)\end{array}$ \\
\hline Earth orientation cabin & 0 & 13.0 \\
Antenna & passive & passive \\
East cabin & 6.0 & 20.0 \\
West cabin & 6.0 & 20.0 \\
South cabin & 4.0 & 30.0 \\
North cabin & -4.0 & 0 \\
Solar wing & 40.0 & 42.0 \\
\hline
\end{tabular}

Based on the Equation (7) and the material parameters, the surface temperature is calculated and showed in the Table 3. Then the IR irradiance is calculated using the IR radiometric module and showed in the Figure 4.

Table 3. The temperature of each cabin surface and the solar wing.

\begin{tabular}{c|cc}
\hline position & $\begin{array}{c}\text { The worst cold case } \\
\left({ }^{\circ} \mathbf{C}\right)\end{array}$ & $\begin{array}{c}\text { The worst hot case } \\
\left({ }^{\circ} \mathbf{C}\right)\end{array}$ \\
\hline Earth orientation cabin & 4.4 & 11.3 \\
Antenna & -11.2 & -7.8 \\
East cabin & 2.6 & 16.5 \\
West cabin & -46 & -35.1 \\
South cabin & 4.4 & 30.3 \\
North cabin & -3.8 & 0.6 \\
Solar wing & 40 & 42 \\
\hline
\end{tabular}

In order to simplify the simulator structure, only the thermal control paint is used as the surface material. And the equivalent surface temperature is designed to get the same IR irradiance as the Figure 4 showed. Then the surface temperature of the simulator is calculated using the reversing progress of the IR irradiance calculation and showed in the Table 4.

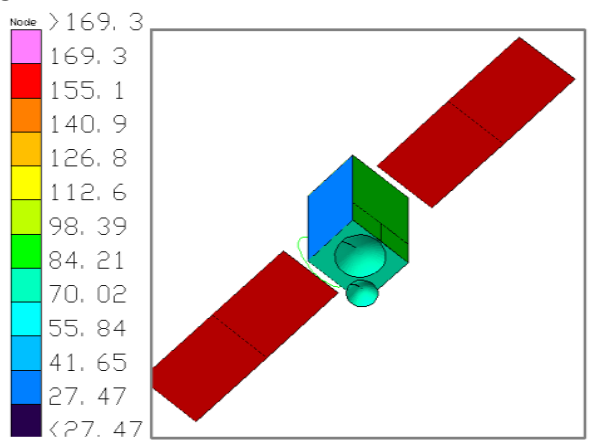

(a) The worst cold case

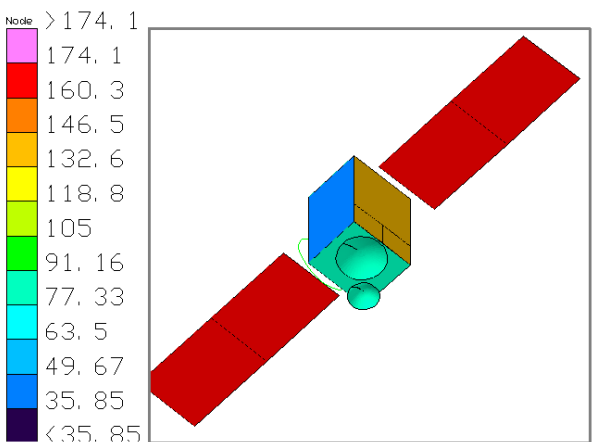

(b) The worst hot case

Figure 4. The surface temperature.

The IR characteristic of the simulator is measured in the cryogenic vacuum chamber, and the heater band and thermocouple are used to control the temperature of the simulator. Liquid nitrogen is used to provide the cryogenic environment in the chamber. 
Table 4. The temperature of the simulator surface.

\begin{tabular}{c|cc}
\hline position & $\begin{array}{c}\text { The worst cold case } \\
\left({ }^{\circ} \mathbf{C}\right)\end{array}$ & $\begin{array}{c}\text { The worst hot case } \\
\left({ }^{\circ} \mathbf{C}\right)\end{array}$ \\
\hline Earth orientation cabin & -9.7 & -3.46 \\
Antenna & -11.2 & -7.8 \\
East cabin & -11.4 & 1.25 \\
West cabin & -55.8 & -45.8 \\
South cabin & -0.97 & 24.0 \\
North cabin & -8.9 & -4.6 \\
Solar wing & 37.8 & 37.0 \\
\hline
\end{tabular}

\section{Discussion and conclusion}

In the work, an IR characteristic model for HEO target is represented. Unlike the traditional model, the temperature of the cabin inner is used as the origin of the simulation. In this way, the input of the modelling could be achieved more practical. 3DS MAX software is used to build the 3D-configuration, so that the model can fit for different target demand. Moreover, the coordinate of each facet is used to calculate the shelter relation among the facets. In the model, some radiation contribution to the target is simplified to decrease the calculation quantity. The irradiance for different temperature implies that the simplified action is valid. Moreover, the simplified action brings in other advantage that the model could be used to calculate the IR radiation of the moving target real-time. Using the model, a HEO target simulator is designed, and the equivalent surface temperature is used to simplify the simulator structure. In the future work, the simulator IR characteristic will be measured in the cryogenic vacuum chamber to evaluate the capability. And the results are also used to evaluate the accuracy of the model further.

\section{References}

1. Y. Zheng, Z. Wei, X. Huizhong, Infrared and Laser Engineering, 37 (2008)

2. W. Jun, H. Yu, D. Na, S. Kefeng, S. Dandan, X. Junhu, W. Wenli, G. Jiaobo, Proceedings of SPIE, 9449 (2015)

3. G. Sili, T. Xinyi, Y. Yang, X. Fengting, Proceedings of SPIE, 7383 (2009)

4. W. Ying, H. Jianming, W. Xiangquan, Infrared and Laser Engineering, 449 (2015)

5. T. Donging, J. Guorui, Z. Huijie, International Journal of Remote Sensing, 3520 (2014)

6. F. Jianying, W. Yang, G. Guanglei, Journal of Harbin University of Science and Technology, 14 6 (2009)

7. S. Chengming, Y. Yan, Z. Xiubao. Proceedings of SPIE, 7383 (2009)

8. M. J. Duggin, C. J. Robinove, International Journal of Remote Sensing, 11 (1990) 